\title{
Evolution of the 5 January 2005 CMEs associated with eruptive filaments in inner heliosphere
}

\author{
Rahul Sharma ${ }^{1}$, Nandita Srivastava ${ }^{2}$, Bernard V. Jackson ${ }^{3}$, \\ D. Chakrabarty ${ }^{4}$, Nolan Luckett ${ }^{3}$, Hsiu-Shan $\mathbf{Y u}{ }^{3}$, Qiang $\mathbf{H u}^{5,6}$ \\ and Christian Möstl ${ }^{7,8,9}$ \\ ${ }^{1} 3$, Indra Nagar, North Sunderwas, Udaipur, India. \\ email: sharmarahul20@googlemail.com
}

${ }^{2}$ Udaipur Solar Observatory, Physical Research Laboratory, Udaipur, India. ${ }^{3}$ Center for Astrophysics and Space Sciences, University of California at San Diego, CA, USA. ${ }^{4}$ Space and Atmospheric Sciences Division, Physical Research Laboratory, Ahmedabad, India.

${ }^{5}$ Department of Physics, The University of Alabama in Huntsville, Huntsville, AL, USA.

${ }^{6}$ Center for Space Plasma and Aeronomic Research, The University of Alabama in Huntsville, Huntsville, AL, USA. ${ }^{7}$ Space Sciences Laboratory, University of California, Berkeley, CA, USA. ${ }^{8}$ Kanzelhöhe Observatory-IGAM, Institute of Physics, University of Graz, Graz, Austria. ${ }^{9}$ Space Research Institute, Austrian Academy of Sciences, A-8042 Graz, Austria.

\begin{abstract}
On 5 January 2005, SoHO/LASCO observed two CMEs associated with eruptive filaments with different initial velocities and acceleration. The second CME accelerates much faster than the previous and the resulting interaction has been revealed in in-situ spacecraft measurements by the presence of magnetic holes at the border of the two distinct magnetic clouds. At their interface region, these magnetic clouds have embedded filament plasma that shows complex magnetic structures with a distinct magnetic flux rope configuration; these have been modeled by the Grad - Shafranov reconstruction technique. The geomagnetic consequences of these structures have been associated with substorms in recovery phase of a storm and detailed analysis is presented in Sharma et al. (2013). In the present paper, we highlight the comparison of shape and extent of two filament plasma remnants in magnetic clouds as revealed by three - dimensional (3D) reconstruction and analysis from the Solar Mass Ejection Imager (SMEI) data. The results provide an overview of the two eruptive filaments on 5 January 2005 and their interplanetary propagation.
\end{abstract}

Keywords. Solar filaments, ICMEs, geomagnetic storms, substorms

\section{Introduction}

Despite the fact that around $70 \%$ of the Coronal Mass Ejections (CMEs) in interplanetary medium are associated with eruptive filaments (Webb \& Hundhausen, 1987), there remain a number of questions concerning identification of their remnants in in-situ data and propagation patterns in the heliosphere. As the CME propagates, the associated magnetic fields may reconnect with solar wind fields or those of other CMEs creating complicated signatures in spacecraft data. Also, CMEs kinematically elongate in angular extent, expand from high internal pressure, and may distort due to inhomogeneous ambient solar wind environment. We focus on the evolution of two CMEs associated with eruptive filaments on 5 January 2005, in the inner heliosphere with identification of filament remnants. The angular extents and general shapes of the associated structures were reconstructed using SMEI data while the magnetic topology of embedded flux ropes is modeled using the Grad-Shafranov reconstruction technique. 


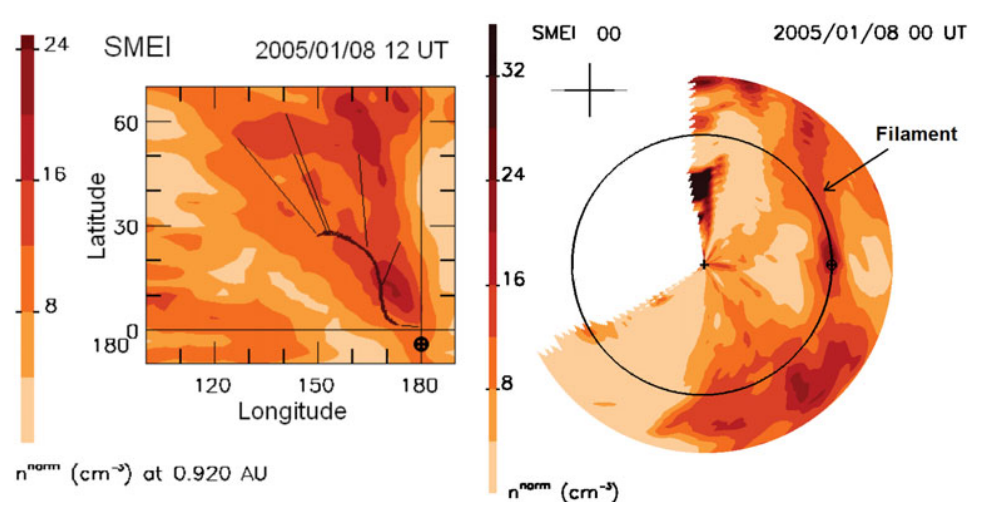

Figure 1. Left: Portions of the filament shown expanded to the larger size in the SMEI reconstructions by black thin lines traced from the surface filament to the expanded structure at 1 AU. Right: 3D reconstructed ecliptic cuts viewed from the north with the Sun at the center and Earth $(\oplus)$ shown to the right on its elliptical orbit.

\section{Data, Analysis and Results}

Solar disk observations were taken from ground-based (KSO and YNAO) and spaceborne (SoHO-EIT/MDI/LASCO) instruments. The in-situ properties were investigated through measurements from ACE and Wind spacecraft. 3D reconstruction of the plasma structures and their extents were done using SMEI data. Filament plasma remnants were identified in magnetic clouds by techniques described by Sharma \& Srivastava (2012). The magnetic topology of embedded magnetic flux ropes is modeled using Grad-Shafranov reconstruction technique (Hu \& Sonnerup, 2002). 3D rendering of associated plasma structures is done by the use of time dependent reconstruction algorithm (Jackson et al., 2002, and references therein).

Sharma et al. (2013) reported the event by the study of remote and in-situ observations of two CMEs associated with an active region and quiescent filaments on 5 January 2005. The first CME was associated with an east - west oriented filament and was slow in velocity and acceleration as compared to the second north - south oriented filament. Due to this difference, the two CMEs interacted in the interplanetary medium through magnetic reconnection which resulted in complex magnetic structures at the interface region, which was further confirmed by modeling of magnetic configuration of flux rope structures using G-S technique. The in-situ signatures of solar wind for the events studied also provide evidence for filament plasma remnants.

3D reconstructions from SMEI data (Fig. 1) reveal that the filament structure(s) near $1 \mathrm{AU}$ have expanded in their latitude and longitude extent on the solar surface to nearly twice the size and volume at 1 AU. We identify the CME associated with first filament eruption to the west and the in-situ remnant as a knotted structure enveloped in the cloud nearer to the Earth. The second filament is observed as a dense structure extending to the northwest to a distance over half an $\mathrm{AU}$ from the Earth. A qualitative comparison with in-situ data suggests that spacecraft (ACE/Wind) encountered a dense, flank edge of the filament structures, associated with 5 January 2005 solar eruptions.

\section{References}

Hu, Q. \& Sonnerup, B. U.Ö. 2002, J. Geophys. Res., 107, 1142

Jackson, B. V., Hick, P. P., \& Buffington, A. 2002, Proc. SPIE, 4853, 23

Sharma, R. \& Srivastava, N. 2012, J. Space Weather Space Clim., 2, A10

Sharma, R., Srivastava, N., Chakrabarty, D., Möstl, C., \& Hu, Q. 2013, J. Geophys. Res., 118

Webb, D. F. \& Hundhausen, A. J. 1987, Sol. Phys., 108, 383 